\title{
Características associadas ao uso de serviços odontológicos públicos pela população adulta brasileira
}

\author{
Characteristics associated with the use of dental services \\ by the adult Brazilian population
}

Rafaela da Silveira Pinto ${ }^{1}$

Divane Leite Matos ${ }^{2}$

Antônio Ignácio de Loyola Filho ${ }^{3}$

${ }^{1}$ Diretoria de Saúde Bucal, Secretaria de Estado de Saúde de Minas Gerais. Cidade Administrativa do Estado de Minas Gerais, Prédio Minas 12\%/Ala par, Serra Verde. 31630-901 Belo Horizonte MG. rafaela.silveira@ saude.mg.gov.br ${ }^{2}$ Prefeitura Municipal de Contagem

${ }^{3}$ Laboratório de Epidemiologia e Antropologia Médica, Centro de Pesquisas René Rachou, Fundação Oswaldo Cruz

\begin{abstract}
The scope of this study was to investigate the factors related to the use of dental services by Brazilian adults. Data were collected from 13,356 adults (35 to 44 years of age), participating in a nationwide epidemiological survey of oral health (SB-BRASIL 2003 Project). Data analysis was based on Poisson regression, which produced estimates of Prevalence Ratios as a measure of association. Data analysis showed that the use of dental services by adults was associated with: female gender, low education and income, living in the Northeastern and Southern regions and in small cities, complaints of toothache or gum pain, need for partial/total prosthesis, a greater amount of permanent teeth requiring treatment, demand for service due to some dental problems and evaluation of dental care received on a regular basis. These results showed that the population attended by the public service was socio-economically less privileged and had greater need for treatment. This situation reflects an historical abandonment of the adult population by the dental healthcare system in Brazil and poses a major challenge to the Unified Health Service, in light of its intended role to reduce inequalities and provide universal access to comprehensive care.
\end{abstract}

Key words Dental health service use, Brazilian adult oral health
Resumo O objetivo deste estudo foi investigar os fatores associados ao uso de serviços odontológicos públicos pela população adulta brasileira. Foram utilizados dados coletados junto a 13.356 adultos (35-44 anos), em um inquérito epidemiológico de saúde bucal, de abrangência nacional (Projeto SBBrasil 2003). A análise dos dados baseou-se na regressão de Poisson, que produziu estimativas de Razões de Prevalências como medida de associação. A análise dos dados mostrou que o uso de serviços odontológicos públicos por adultos esteve associada com: sexo feminino, baixa escolaridade e renda, residência nas regiões Nordeste e Sul e em municípios de menor porte, queixas de dor de dente ou gengival, necessidades de prótese parcial e total, maior quantidade de dentes permanentes demandando tratamento, procura por serviço motivada por algum problema bucal e avaliação do tratamento recebido como regular. Esses resultados mostraram que a população atendida pelo serviço público era menos favorecida socioeconomicamente e apresentava maiores necessidades de tratamento. A grande necessidade acumulada reflete o histórico apartamento desse segmento populacional da atenção à saúde bucal em nosso país e coloca um grande desafio ao SUS, considerando o seu papel na redução das desigualdades e de provimento de acesso universal ao cuidado integral.

Palavras-chave Uso de serviços odontológicos, Saúde bucal, Adulto, Brasil 


\section{Introdução}

Historicamente, a atenção odontológica pública no Brasil priorizou as crianças matriculadas em escola como população-alvo e focou suas ações na abordagem da cárie dentária ${ }^{1,2}$. Esse privilégio ocorreu em detrimento de outros segmentos populacionais, como a dos adultos, que conviveram com um modelo público que limitava a atenção à população inserida no mercado formal de trabalho, e era calcado na demanda espontânea, no predomínio de ações curativas, de caráter mutilador. Esse modelo tinha o Estado como grande financiador e o setor privado como principal prestador de serviços ${ }^{3}$.

O quadro de saúde bucal dos adultos, resultante desse processo, é pouco animador e não apresentou alterações importantes nas duas últimas décadas do século passado, como revelam os inquéritos de abrangência nacional que avaliaram a saúde bucal do adulto brasileiro, realizados em 1986 e $2003^{4}$. Os adultos brasileiros apresentavam, em 2003, CPO-D médio (índice de saúde bucal que traduz a experiência de cárie do indivíduo ao longo da vida) superior a 20 dentes, sendo o componente que representa os dentes perdidos (sem possibilidade de recuperação) superior a $60 \%$. Além disso, um percentual baixo dessa população (próximo a 22\%) apresentava periodonto saudável (sem alterações como sangramento gengival, cálculo ou bolsa periodontal) $)^{5}$.

Estudos sobre a utilização de serviços de saúde são considerados importantes, por permitirem caracterizar a população usuária, identificar suas condições de saúde e as suas motivações para a procura, aspectos fundamentais no planejamento e na organização das ações de saúde. Andersen e Newman ${ }^{6}$ construíram um modelo para explicar o uso de serviços de saúde, que agrega fatores associados aos determinantes sociais, individuais, relacionados aos sistemas e serviços de saúde, e à utilização dos últimos. Em relação aos determinantes individuais, o modelo considera três categorias: (1) predisponentes, relacionados à tendência do indivíduo utilizar o serviço; (2) capacitantes, que contribuem para tornar os recursos dos serviços de saúde disponíveis ao indivíduo; e (3) os de necessidade, que dizem respeito às condições de saúde percebidas pelo usuário ou definidas pelo profissional de saúde. Este modelo tem sido empregado em estudos internacionais ${ }^{7,8}$ e nacionais ${ }^{9,10}$ sobre a utilização de serviços odontológicos, abordando diferentes segmentos populacionais e tendo como foco a comparação entre usuários e não usuários.
Estudos brasileiros sobre a utilização de serviços odontológicos, especificamente entre adultos, são raros e voltaram-se para a investigação de características associadas ao uso regular de serviços ${ }^{10} \mathrm{e}$ à dor de dente como motivo de consulta $^{11}$. O presente trabalho utilizou o banco de dados do Projeto SB-Brasil $2003^{12}$ para investigar as características associadas ao uso de serviços odontológicos públicos por adultos, tendo como referencial o modelo comportamental de Andersen e Newman ${ }^{6}$.

\section{Metodologia}

\section{Fonte de dados}

O presente estudo utilizou o banco de dados do Projeto SB Brasil 2003 - Condições de Saúde Bucal da População Brasileira ${ }^{12}$, um inquérito epidemiológico que investigou os aspectos tais como os principais agravos de saúde e a utilização de serviços odontológicos, entre outros. O inquérito abordou uma amostra representativa da população brasileira para as grandes regiões geográficas, para cada tipo de município (porte populacional) e para as idades de 5 e de 12 anos, e para as faixas etárias de 18 a 36 meses, 15 a 19 anos, 35 a 44 anos e 65 a 74 anos.

A seleção de participantes foi feita por meio de amostra probabilística por conglomerados, obtida em três estágios de seleção: os municípios foram as unidades primárias, os setores censitários foram as unidades secundárias e as quadras e domicílios foram as unidades terciárias. Para a faixa etária dos adultos, todos os moradores dos domicílios selecionados que concordaram em participar foram entrevistados e examinados.

Os dados foram coletados por meio de exames clínicos e entrevistas, realizados por um cirurgião-dentista e auxiliar, devidamente treinados e calibrados. A concordância dos exames clínicos intra e inter examinadores foi considerada $\mathrm{boa}^{13}$. Maiores detalhes podem ser vistos em outras publicações s2,13. $^{12}$.

No presente estudo analisaram-se os dados relativos à população adulta (35-44 anos). Segundo a Organização Mundial de Saúde ${ }^{14}$, utilizar esta faixa etária em inquéritos epidemiológicos permite verificar o efeito total da cárie dentária, a gravidade do envolvimento periodontal e os efeitos do tratamento prestado, permitindo assim, uma boa aferição das condições de saúde bucal em adultos. 


\section{Variáveis de estudo}

Aos participantes foi indagado (1) se haviam ido ao dentista alguma vez na vida e, em caso afirmativo, (2) o tipo de serviço utilizado. Foram selecionados para o estudo todos os adultos que responderam afirmativamente à primeira pergunta. A variável dependente foi a utilização de serviços odontológicos públicos, construída a partir das respostas à segunda pergunta. Os participantes que responderam "público" compuseram o grupo-evento, e aqueles que relataram ter utilizado um serviço odontológico privado liberal, ou vinculado a plano de saúde e convênios, constituíram o grupo de referência.

A seleção das variáveis independentes foi baseada no modelo comportamental de Andersen e Newman ${ }^{6}$, idealizado para as investigações sobre a utilização de serviços de saúde. Os fatores predisponentes considerados foram: sexo (masculino vs feminino), idade (35-39 anos vs 40-44 anos) e escolaridade, medida em função do número de anos de frequência à escola regular $(\geq 9$; 5-8; 1-4 e nenhum). Os fatores capacitantes foram: a renda per capita, medida em Reais $(\leq 50$; 51-100; 101-200 e $\geq 201$ ), grandes regiões geográficas (Sudeste, Norte, Nordeste, Sul e CentroOeste), local de residência (urbano vs rural) e porte do município, em função da população residente (<10.000; 10.000-100.000 e $>100.000)$.

Entre os fatores de necessidades (de saúde e de tratamento) considerou-se separadamente aquelas necessidades percebidas pelo respondente e aquelas definidas pelo profissional ao exame clínico. No primeiro caso, foram investigadas a auto-avaliação da saúde bucal (ótima/boa; regular; ruim/péssima), necessidade de tratamento auto-referida (não vs sim) e queixa de dor de dente/gengival (não vs sim). Já as necessidades definidas pelo profissional incluíram necessidade prótese (não; sim-parcial; sim-total), presença de alteração de tecido mole (não vs sim), número de dentes com necessidade de tratamento (nenhum; 1-3; 4 ou mais; não se aplica), presença de problema periodontal (não; cálculo/sangramento; bolsa e outros).

No caso da variável "necessidade de prótese", foi considerado sem necessidade o indivíduo com todos os dentes presentes, ou com espaços protéticos presentes com prótese ou ainda desdentado total com presença de prótese. Já a categoria "sim, parcial” são aqueles indivíduos que necessitavam de próteses fixas ou removíveis para substituição de um ou mais elementos ou ainda a combinação de próteses. A categoria "sim, to- tal" é representada pelos indivíduos desdentados totais que não usavam prótese no momento. A categoria "não se aplica", da variável número de dentes com necessidade de tratamento, refere-se aos edentados, e a categoria "outros" da variável presença de problema periodontal se refere a sextantes excluídos, que são aqueles com menos de dois dentes presentes, o que inviabilizou a aferição do índice utilizado (Índice Periodontal Comunitário - CPI). Em ambos os casos, a manutenção dessas categorias se deu com o objetivo de preservar a matriz de análise, sem prejuízo do poder estatístico da amostra.

As variáveis independentes consideradas para a descrição das características de utilização dos serviços odontológicos foram o motivo da visita ao dentista (rotina; problemas bucais e outros), recebimento de informação sobre a saúde bucal na visita ao dentista (não vs sim) e avaliação do atendimento recebido (bom/ótimo; regular; ruim/péssimo). A construção das categorias da variável motivo da consulta ao dentista foi feita da seguinte forma: considerou-se "consulta de rotina" aquela que o usuário relatou ter procurado o serviço para manutenção ou reparos; já a consulta por problemas bucais foram aquelas motivadas por dor, sangramento gengival, cavidades nos dentes, feridas, caroços ou manchas na boca; e na categoria "outros" foram incluídos os motivos de procura que não se enquadraram nas categorias anteriores.

\section{Análise dos dados}

A análise dos dados baseou-se no modelo hierarquizado, proposto por Victora et al. ${ }^{15}$. Este modelo é mais seletivo e prioriza o conhecimento dos determinantes sociais e biológicos do evento sob investigação, de tal forma que a seleção das variáveis não seja dependente apenas de critérios estatísticos. A análise se baseia numa estrutura conceitual em que as variáveis se interrelacionam em níveis hierárquicos, definidos em função da maior distância (variáveis distais) ou proximidade (variáveis proximais) do evento. Assim é que as variáveis distais (nível hierárquico superior) têm seu efeito mediado pelas variáveis proximais (nível hierárquico inferior); por outro lado, as primeiras constituem potenciais fatores de confusão para o efeito das últimas ${ }^{15}$.

As variáveis foram agrupadas em blocos hierarquizados, do nível mais distal para o mais proximal, a saber: fatores predisponentes (nível 1), fatores capacitantes (nível 2), fatores de necessidade de saúde e de tratamento (percebidas 
pelo respondente e definidas pelo profissional ao exame) (nível 3) e fatores relacionados às características de utilização dos serviços odontológico (nível 4).

A medida de associação utilizada foi a Razão de Prevalências, com respectivo intervalo de confiança de $95 \%$, estimadas por meio da regressão de Poisson. Numa primeira etapa, foram realizadas as análises univariadas, para configurar a descrição das características da população de estudo e a produção de razões de prevalências brutas (com respectivos intervalos de confiança de 95\%) para associações entre as covariáveis e a variávelevento. Em seguida, foram realizadas as análises multivariadas, considerando os blocos de variáveis estruturados com base no modelo de Andersen e Newman ${ }^{6}$, e o sequenciamento proposto pelo modelo de análise hierarquizada adotado. Inicialmente, foi construído o modelo relativo ao bloco de variáveis do nível hierárquico mais distal (nível 1, dos fatores predisponentes), seguindose a inclusão dos demais blocos de variáveis, conforme a sequência dos níveis já descrita.

O critério de significância estatística adotado foi valor de $\mathrm{p}<0,05$, utilizado para: (1) definição das variáveis de um bloco a serem mantidas no modelo referente ao nível hierárquico subsequente; (2) identificação das variáveis do modelo final (nível 4) que permaneceram independentemente associadas ao evento sob investigação. A análise dos dados foi realizada utilizando-se o programa Stata (Stata Corp. College Station, Estados Unidos), versão 9.0.

\section{Aspectos Éticos}

Todos os participantes do estudo receberam uma carta explicando os objetivos e procedimentos a serem realizados e assinaram um Termo de Consentimento Livre e Esclarecido. O projeto SBBrasil foi aprovado, e registrado no CONEP.

\section{Resultados}

Foram selecionados para o estudo 13.356 adultos que responderam ao questionário e submeteram-se a exames clínicos. Desses, foram excluídos 378 que relataram nunca ter ido ao dentista ao longo da vida. Como o estudo restringiu a investigação aos usuários de serviços odontológicos classificados como público, privado liberal ou privado plano de saúde, foram excluídos também 553 adultos que afirmaram ter utilizado serviços do tipo filantrópicos ou serviços rotulados no questionário como "outros", de tipificação desconhecida. Esta investigação baseou-se na análise dos dados relativos a 12.425 adultos com idade entre 35-44 anos.

Em sua maioria, os participantes eram do sexo feminino $(68,2 \%)$, com idade média de 39,2 $(\mathrm{dp}=3,2)$ anos; $40,6 \%$ apresentaram escolaridade de no máximo 4 anos (6,8\% nunca frequentaram a escola), 56,0\% tinham renda familiar per capita de no máximo $\mathrm{R} \$ 120,00$ (equivalente a meio salário mínimo vigente á época do inquérito) e aproximadamente 9 em 10 residiam na zona urbana. A prevalência do uso de serviços públicos foi de $51,8 \%$, enquanto $37,1 \%$ relataram uso de serviço privado liberal e 11,2\% fizeram uso de planos de saúde ou convênios.

$\mathrm{Na}$ Tabela 1 estão descritas as características predisponentes, capacitantes, de condições de saúde e necessidades de tratamento e do uso de serviços de saúde, em função do tipo de serviço utilizado. Nelas encontram-se também os resultados das análises univariadas, em termos das medidas de associação e respectivos intervalos de confiança de $95 \%$.

No tocante às características predisponentes, verifica-se que a prevalência do uso de serviço odontológico público foi maior entre as mulheres $(53,3 \%)$ e menor na faixa etária de 40-44 anos (50,5\%). Em relação à escolaridade, o uso de serviço público aumentou à medida que a escolaridade diminuía, sendo que os adultos sem escolaridade utilizaram aproximadamente duas vezes mais o serviço público do que aqueles com a escolaridade igual ou maior que 9 anos. Na análise univariada, todas as características predisponentes mostraram-se significativamente associadas ao uso de serviço público $(\mathrm{p}<0,05)$ (Tabela 1$)$.

$\mathrm{Na}$ análise dos fatores capacitantes, os adultos de maior renda e residentes em municípios de maior porte utilizaram menos frequentemente os serviços públicos: a prevalência de utilização dos serviços foi de $72,2 \%$ entre os adultos de menor faixa de renda, caindo para $26,3 \%$ entre aqueles de maior renda; ela foi de 59,9\% entre os adultos residentes em municípios de pequeno porte ( $<10$ mil habitantes), decrescendo para $42,2 \%$ entre os residentes em municípios de grande porte (população >100 mil). Já entre os moradores da zona rural, o uso de serviço público foi $20 \%$ superior ao verificado entre os residentes em zona urbana. Essas variáveis apresentaram associações significativas na análise univariada. Com relação às regiões geográficas, os indivíduos das regiões Norte, Nordeste e Sul utilizaram mais o serviço público comparado aos resi- 
Tabela 1. Distribuição dos usuários de serviços odontológicos segundo fatores predisponentes, capacitantes, necessidade de tratamento (autorreferida e diagnosticada pelo profissional) e características da utilização do serviço, Projeto SB-BRASIL 2003.

\begin{tabular}{|c|c|c|c|c|c|}
\hline \multirow[b]{2}{*}{ Variáveis } & \multicolumn{2}{|c|}{ Uso de serviço público } & \multicolumn{2}{|c|}{ Uso de serviço privado } & \multirow[b]{2}{*}{ RP (IC 95\%) } \\
\hline & $\mathrm{N}$ & $\%$ & $\mathrm{~N}$ & $\%$ & \\
\hline \multicolumn{6}{|l|}{ Fatores Predisponentes } \\
\hline \multicolumn{6}{|l|}{ Sexo } \\
\hline Masculino & 1.912 & 48,4 & 2.035 & 51,6 & 1,0 \\
\hline Feminino & 4.520 & 53,3 & 3.958 & 46,7 & $1,10(1,06-1,15)$ \\
\hline \multicolumn{6}{|l|}{ Idade } \\
\hline 35-39 & 3.569 & 52,9 & 3.184 & 47,2 & 1,0 \\
\hline 40-44 & 2.863 & 50,5 & 2.809 & 49,5 & $0,96(0,92-0,99)$ \\
\hline \multicolumn{6}{|l|}{ Escolaridade } \\
\hline$\geq 9$ anos & 1.183 & 35,3 & 2.172 & 64,7 & 1,0 \\
\hline $5-8$ & 1.956 & 51,5 & 1.842 & 48,5 & $1,46(1,36-1,57)$ \\
\hline $1-4$ & 2.636 & 62,9 & 1.557 & 37,1 & $1,78(1,64-1,94)$ \\
\hline nenhuma & 586 & 69,1 & 262 & 30,9 & $1,96(1,77-2,17)$ \\
\hline \multicolumn{6}{|l|}{ Fatores Capacitantes } \\
\hline \multicolumn{6}{|c|}{ Renda per capita (em Reais) } \\
\hline Até 50 & 2.180 & 72,2 & 840 & 27,8 & 1,0 \\
\hline $51-100$ & 2.003 & 61,5 & 1.253 & 38,5 & $0,85(0,81-0,89)$ \\
\hline $101-200$ & 1.452 & 45,6 & 1.736 & 54,5 & $0,63(0,59-0,67)$ \\
\hline$\geq 201$ & 750 & 26,3 & 2.107 & 73,8 & $0,36(0,33-0,40)$ \\
\hline \multicolumn{6}{|l|}{ Regiões geográficas } \\
\hline Sudeste & 1.018 & 45,9 & 1.199 & 54,1 & 1,0 \\
\hline Nordeste & 1.790 & 60,3 & 1.180 & 39,7 & $1,31(1,12-1,54)$ \\
\hline Norte & 1.361 & 60,1 & 902 & 39,9 & $1,31(1,10-1,56)$ \\
\hline Sul & 1.447 & 48,0 & 1.567 & 52,0 & $1,05(0,87-1,26)$ \\
\hline Centro-Oeste & 816 & 41,6 & 1.145 & 58,4 & $0,91(0,75-1,10)$ \\
\hline \multicolumn{6}{|l|}{ Local de residência } \\
\hline Urbano & 5.573 & 50,6 & 5.439 & 49.4 & 1,0 \\
\hline Rural & 855 & 60,8 & 552 & 39,2 & $1,20(1,07-1,34)$ \\
\hline \multicolumn{6}{|l|}{ Porte do município } \\
\hline$<10$ mil & 2.402 & 59,9 & 1.608 & 40,1 & 1,0 \\
\hline 10-100 mil & 2.820 & 50,8 & 2.728 & 49,2 & $0,85(0,76-0,94)$ \\
\hline$>100 \mathrm{mil}$ & 1.210 & 42,2 & 1.657 & 57,8 & $0,71(0,62-0,80)$ \\
\hline \multicolumn{6}{|c|}{ Fatores de necessidade de tratamento } \\
\hline \multicolumn{6}{|c|}{$\begin{array}{l}\text { Necessidade de tratamento } \\
\text { autorreferida }\end{array}$} \\
\hline \multicolumn{6}{|c|}{ Percepção de necessidade } \\
\hline Não & 1.137 & 44,6 & 1.410 & 55,4 & 1,0 \\
\hline Sim & 5.274 & 53,6 & 4.570 & 46,4 & $1,20(1,11-1,30)$ \\
\hline \multicolumn{6}{|l|}{ Autoavaliação de saúde } \\
\hline Ótimo/bom & 2.258 & 46,8 & 2.572 & 53,3 & 1,0 \\
\hline Regular & 2.452 & 52,6 & 2.209 & 47,4 & $1,13(1,07-1,19)$ \\
\hline Ruim/Péssimo & 1.543 & 58,0 & 1.117 & 42,0 & $1,24(1,16-1,33)$ \\
\hline \multicolumn{6}{|c|}{ Queixa de dor de dente/ gengival } \\
\hline Não & 3.953 & 48,8 & 4.152 & 51,2 & 1,0 \\
\hline Sim & 2.478 & 57,4 & 1.841 & 42,6 & $1,18(1,12-1,23)$ \\
\hline \multicolumn{6}{|c|}{$\begin{array}{l}\text { Necessidade de tratamento } \\
\text { diagnosticada pelo profissional }\end{array}$} \\
\hline \multicolumn{6}{|c|}{ Necessidade de prótese } \\
\hline Não & 1.239 & 39,5 & 1.897 & 60,5 & 1,0 \\
\hline Sim, parcial & 4.759 & 54,8 & 3.922 & 45,2 & $1,39(1,30-1,49)$ \\
\hline Sim, total & 389 & 71,8 & 153 & 28,2 & $1,82(1,66-1,99)$ \\
\hline
\end{tabular}


Tabela 1. continuação

\begin{tabular}{|c|c|c|c|c|c|}
\hline \multirow[b]{2}{*}{ Variáveis } & \multicolumn{2}{|c|}{ Uso de serviço público } & \multicolumn{2}{|c|}{ Uso de serviço privado } & \multirow[b]{2}{*}{ RP (IC 95\%) } \\
\hline & $\mathrm{N}$ & $\%$ & $\mathrm{~N}$ & $\%$ & \\
\hline Presença de alteração de tecido mole & & & & & 1,0 \\
\hline Não & 5.629 & 51,6 & 5.275 & 48,4 & $1,02(0,93-1,11)$ \\
\hline $\operatorname{Sim}$ & 759 & 52,4 & 689 & 47,6 & \\
\hline $\begin{array}{l}\text { Total de dentes necessitando de } \\
\text { tratamento }\end{array}$ & & & & & 1,0 \\
\hline Nenhum & 1.494 & 42,4 & 2.026 & 57,6 & $1,22(1,13-1,31)$ \\
\hline $1-3$ & 2.185 & 51,6 & 2.051 & 48,4 & $1,43(1,30-1,57)$ \\
\hline 4 ou mais & 2.135 & 60,5 & 1.394 & 39,5 & $1,28(1,15-1,41)$ \\
\hline Não se aplica & 618 & 54,2 & 522 & 45,8 & \\
\hline Problemas periodontais & & & & & 1,0 \\
\hline Hígido & 1.267 & 45,4 & 1.522 & 54,6 & $1,18(1,09-1,27)$ \\
\hline Cálculo/Sangramento & 3.750 & 53,6 & 3.248 & 46,4 & $1,13(1,00-1,27)$ \\
\hline Bolsa & 632 & 51,3 & 600 & 48,7 & $1,21(1,10-1,34)$ \\
\hline Outros (sextante excluído) & 572 & 55,2 & 465 & 44,8 & \\
\hline \multicolumn{6}{|l|}{ Características da utilização do serviço } \\
\hline Motivo da visita & & & & & 1,0 \\
\hline Consulta de rotina & 1.286 & 37,7 & 2.125 & 62,3 & $1,56(1,42-1,71)$ \\
\hline Problemas bucais & 4.764 & 58,7 & 3.353 & 41,3 & $1,13(0,99-1,30)$ \\
\hline Outros & 382 & 42,6 & 515 & 57,4 & \\
\hline $\begin{array}{l}\text { Recebimento de informação sobre } \\
\text { saúde bucal }\end{array}$ & & & & & 1,0 \\
\hline Não & 2.997 & 56,4 & 2.317 & 43,6 & $0,86(0,81-0,91)$ \\
\hline $\operatorname{Sim}$ & 3.433 & 48,3 & 3.673 & 51,7 & \\
\hline Avaliação do atendimento recebido & & & & & 1,0 \\
\hline Bom/Ótimo & 5.366 & 50,4 & 5.286 & 49,6 & $1,22(1,14-1,30)$ \\
\hline Regular & 726 & 61,5 & 455 & 38,5 & $1,14(1,00-1,29)$ \\
\hline Ruim/Péssimo & 324 & 57,4 & 241 & 42,7 & \\
\hline
\end{tabular}

RP (IC95\%): Razão de prevalências bruta,(Intervalo de Confiança 95\%), obtidos pela regressão de Poisson.

dentes na região Sudeste, enquanto os residentes na região Centro-Oeste utilizaram menos; todavia, somente as diferenças relativas às regiões Norte e Nordeste foram estatisticamente significativas (Tabela 1).

Os resultados relativos às condições de saúde e necessidades de tratamento (autorreferidas e definidas pelo profissional) também estão descritos na Tabela 1. De modo geral, os adultos em piores condições de saúde utilizaram com maior frequência os serviços públicos. Assim é que o uso deste foi mais frequente entre os adultos que se percebiam com necessidade de tratamento $(53,6 \%)$, que avaliaram sua própria saúde como ruim/péssima $(58,0 \%)$ e que se queixaram de dor de dentes e/ou gengiva $(57,4 \%)$, do que, respectivamente, entre aqueles que não referiram necessidade de tratamento $(44,6 \%)$, que avaliaram sua saúde como ótima ou boa $(46,8 \%)$ e que não referiram queixa de dor de dente e/ou gengiva $(48,8 \%)$. Da mesma forma, os adultos que tiveram detectadas, ao exame profissional, necessidades de tratamento odontológico (presença de alterações de tecido mole, de cálculo/sangramento ou bolsa periodontal, dentes demandando restaurações e necessidade de prótese total ou parcial), utilizaram mais os serviços odontológicos públicos do que aqueles que não sofriam dessas condições. Entre todos os fatores de necessidade, apenas a presença de alterações de tecido mole não esteve significativamente associada ao uso de serviços públicos, na análise univariada.

Ainda conforme a Tabela 1, o uso de serviço odontológico público foi mais frequente entre aqueles que o fizeram motivados por problemas bucais $(58,7 \%$ contra $37,7 \%$ que o fizeram como rotina) e avaliaram mal o atendimento recebido $(61,5 \%$ e $57,3 \%$, respectivamente para regular e 
ruim/péssimo, contra 50,4\% que avaliaram o atendimento recebido como bom/ótimo). Já no que tange ao recebimento de informações sobre como evitar problemas bucais, a maioria das pessoas que não as receberam foi usuária do serviço público $(56,4 \%)$. Todas as variáveis deste bloco apresentaram associações significativas na análise univariada.

As Tabelas 2 a 5 referem-se às análises multivariadas hierarquizadas, e apresentam os resultados em sequência, a partir do nível 1 (características predisponentes, o mais distal) até o nível 4 (características de utilização dos serviços odontológicos, proximal).

De acordo com a análise multivariada do primeiro nível hierárquico, todas as variáveis predisponentes permaneceram significativamente associadas ao uso de serviços odontológicos públicos, sendo que a variável escolaridade foi a mais fortemente associada. Observou-se associação negativa entre esta variável e o uso de serviços odontológicos públicos, que aumentou à medida que a escolaridade diminuía $(\mathrm{RP}=1,47$, IC95\% 1,37-1,58 para 5-8 anos; RP=1,80, IC95\% 1,65-1,96 para 1 -4 anos e $\mathrm{RP}=1,99$; IC95\% 1,802,20 para nenhuma escolaridade). Todas as variáveis foram mantidas para o ajustamento do nível hierárquico subsequente - nível 2 (Tabela 2).

$\mathrm{Na}$ análise do nível 2, relativo aos fatores capacitantes (Tabela 3 ), apenas o local de residência (urbano/rural) deixou de ser estatisticamente significativo $(\mathrm{p}=0,739)$, após o ajustamento mútuo e pelo bloco de variáveis predisponentes.

Tabela 2. Resultados da análise multivariada para as variáveis predisponentes, Projeto SB-BRASIL 2003.

\begin{tabular}{|c|c|c|}
\hline Variáveis & $\begin{array}{c}\text { RP ajustada } \\
\text { IC }(95 \%)\end{array}$ & Valor de $p^{*}$ \\
\hline \multicolumn{3}{|l|}{ Sexo } \\
\hline Masculino & 1,0 & \\
\hline Feminino & $1,11(1,07-1,16)$ & $<0,001$ \\
\hline \multicolumn{3}{|l|}{ Idade } \\
\hline 35-39 & 1,0 & \\
\hline 40-44 & $0,93(0,89-0,96)$ & $<0,001$ \\
\hline \multicolumn{3}{|l|}{ Escolaridade } \\
\hline$\geq 9$ anos & 1,0 & \\
\hline $5-8$ & $1,47(1,37-1,58)$ & $<0,001$ \\
\hline $1-4$ & $1,80(1,65-1,96)$ & $<0,001$ \\
\hline nenhuma & $1,99(1,80-2,20)$ & $<0,001$ \\
\hline
\end{tabular}

*Razão de Prevalências (Intervalo de Confiança 95\%), ajustada por todas as variáveis da tabela. " Valor de p obtido pelo teste de Wald
Entre as demais variáveis capacitantes, a renda familiar per capita e o porte do município permaneceram negativamente associadas ao uso de serviços públicos. Com o ajustamento pelas variáveis predisponentes, além das regiões Norte e Nordeste, a região Sul também passou a apresentar associação significativa com o uso de serviço público ( $\mathrm{RP}=1,18$; IC95\% 1,01-1,37).

Na Tabela 4, são apresentados resultados das análises relativas ao bloco de variáveis que traduzem as necessidades de tratamento e condições de saúde, ajustadas entre si e pelas variáveis dos níveis hierárquicos superiores (predisponentes e capacitantes).

Tabela 3. Resultados da análise multivariada para os fatores capacitantes, ajustados entre si e pelos fatores predisponentes, Projeto SB-BRASIL 2003.

\begin{tabular}{|c|c|c|}
\hline Variáveis & $\begin{array}{l}\text { RP ajustada } \\
\text { IC }(95 \%)\end{array}$ & $\begin{array}{l}\text { Valor } \\
\text { de } p^{\star *}\end{array}$ \\
\hline \multicolumn{3}{|c|}{ Fatores Predisponentes } \\
\hline \multicolumn{3}{|c|}{ Sexo } \\
\hline Masculino & 1,0 & \\
\hline Feminino & $1,07(1,03-1,11)$ & $<0,001$ \\
\hline \multicolumn{3}{|l|}{ Idade } \\
\hline $35-39$ & 1,0 & \\
\hline 40-44 & $0,96(0,93-1,00)$ & 0,023 \\
\hline \multicolumn{3}{|l|}{ Escolaridade } \\
\hline$\geq 9$ anos & 1,0 & \\
\hline $5-8$ & $1,26(1,18-1,35)$ & $<0,001$ \\
\hline $1-4$ & $1,38(1,29-1,49)$ & $<0,001$ \\
\hline Nenhuma & $1,43(1,30-1,57)$ & $<0,001$ \\
\hline \multicolumn{3}{|c|}{ Fatores Capacitantes } \\
\hline \multicolumn{3}{|c|}{ Renda per capita (em Reais) } \\
\hline Até 50 & 1,0 & $<0,001$ \\
\hline $51-100$ & $0,90(0,86-0,94)$ & $<0,001$ \\
\hline $101-200$ & $0,71(0,67-0,76)$ & $<0,001$ \\
\hline$\geq^{\wedge} 201$ & $0,46(0,41-0,50)$ & $<0,001$ \\
\hline \multicolumn{3}{|l|}{ Regiões geográficas } \\
\hline Sudeste & 1,0 & \\
\hline Nordeste & $1,21(1,05-1,39)$ & 0,007 \\
\hline Norte & $1,18(1,02-1,38)$ & 0,030 \\
\hline Sul & $1,18(1,01-1,37)$ & 0,032 \\
\hline Centro-Oeste & $0,96(0,82-1,14)$ & 0,655 \\
\hline \multicolumn{3}{|l|}{ Local de residência } \\
\hline Urbano & 1,0 & \\
\hline Rural & $0,98(0,89-1,09)$ & 0,739 \\
\hline \multicolumn{3}{|l|}{ Porte do município } \\
\hline$<10$ mil & 1,0 & \\
\hline 10-100 mil & $0,91(0,83-1,00)$ & 0,045 \\
\hline$>100 \mathrm{mil}$ & $0,84(0,75-0,95)$ & 0,003 \\
\hline
\end{tabular}

* Razão de Prevalências (Intervalo de Confiança 95\%), ajustada por todas as variáveis da tabela. ${ }^{*}$ Valor de $\mathrm{p}$ obtido pelo teste de Wald 
Tabela 4. Resultados da análise multivariada para as necessidades de tratamento (autorreferidas e diagnosticadas pelo profissional), ajustadas entre si e pelos fatores predisponentes e capacitantes, Projeto SB-BRASIL 2003.

\begin{tabular}{|c|c|c|}
\hline Variáveis & $\begin{array}{l}\text { RP ajustada* } \\
\text { IC }(95 \%)\end{array}$ & $\begin{array}{l}\text { Valor } \\
\text { de } p^{* *}\end{array}$ \\
\hline \multicolumn{3}{|l|}{ Fatores Predisponentes } \\
\hline \multicolumn{3}{|l|}{ Sexo } \\
\hline Masculino & 1,0 & \\
\hline Feminino & $1,07(1,03-1,12)$ & 0,001 \\
\hline \multicolumn{3}{|l|}{ Idade } \\
\hline 35-39 & 1,0 & \\
\hline 40-44 & $0,98(0,94-1,01)$ & 0,153 \\
\hline \multicolumn{3}{|l|}{ Escolaridade } \\
\hline$\geq 9$ anos & 1,0 & \\
\hline $5-8$ & $1,21(1,14-1,29)$ & $<0,001$ \\
\hline $1-4$ & $1,32(1,24-1,41)$ & $<0,001$ \\
\hline Nenhuma & $1,37(1,25-1,50)$ & $<0,001$ \\
\hline \multicolumn{3}{|l|}{ Fatores Capacitantes } \\
\hline \multicolumn{3}{|l|}{ Renda per capita (em Reais) } \\
\hline Até 50 & 1,0 & \\
\hline $51-100$ & $0,91(0,86-0,95)$ & $<0,001$ \\
\hline $101-200$ & $0,73(0,68-0,78)$ & $<0,001$ \\
\hline$\geq^{\wedge} 201$ & $0,47(0,43-0,52)$ & $<0,001$ \\
\hline \multicolumn{3}{|l|}{ Regiões geográficas } \\
\hline Sudeste & 1,0 & \\
\hline Nordeste & $1,19(1,03-1,37)$ & 0,018 \\
\hline Norte & $1,14(0,98-1,33)$ & 0,095 \\
\hline Sul & $1,19(1,02-1,39)$ & 0,023 \\
\hline Centro-Oeste & $0,96(0,81-1,13)$ & 0,589 \\
\hline \multicolumn{3}{|l|}{ Porte do município } \\
\hline$<10$ mil & 1,0 & \\
\hline $10-100 \mathrm{mil}$ & $0,90(0,82-0,99)$ & 0,025 \\
\hline$>100$ mil & $0,83(0,74-0,94)$ & 0,003 \\
\hline \multicolumn{3}{|c|}{ Fatores de necessidade de tratamento } \\
\hline \multicolumn{3}{|c|}{ Necessidade de tratamento autorreferida } \\
\hline \multicolumn{3}{|c|}{ Percepção de necessidade } \\
\hline Não & 1,0 & \\
\hline Sim & $1,04(0,97-1,12)$ & 0,231 \\
\hline \multicolumn{3}{|l|}{ Autoavaliação de saúde } \\
\hline Ótimo/bom & 1,0 & \\
\hline Regular & $1,03(0,98-1,07)$ & 0,234 \\
\hline Ruim/Péssimo & $1,01(0,95-1,07)$ & 0,748 \\
\hline \multicolumn{3}{|l|}{ Queixa de dor de dente/gengival } \\
\hline Não & 1,0 & \\
\hline Sim & $1,07(1,03-1,11)$ & 0,001 \\
\hline \multicolumn{3}{|c|}{$\begin{array}{l}\text { Necessidade de tratamento diagnosticada pelo } \\
\text { profissional }\end{array}$} \\
\hline \multicolumn{3}{|c|}{ Necessidade de prótese } \\
\hline Não & 1,0 & \\
\hline Sim, parcial & $1,20(1,12-1,29)$ & $<0,001$ \\
\hline Sim, total & $1,35(1,24-1,48)$ & $<0,001$ \\
\hline \multicolumn{3}{|c|}{ Presença de alteração de tecido mole } \\
\hline Não & 1,0 & \\
\hline Sim & $0,97(0,90-1,04)$ & 0,359 \\
\hline \multicolumn{3}{|c|}{ Total de dentes necessitando de tratamento } \\
\hline Nenhum & 1,0 & \\
\hline $1-3$ & $1,06(1,00-1,13)$ & 0,057 \\
\hline 4 ou mais & $1,12(1,04-1,21)$ & 0,002 \\
\hline Não se aplica & $1,06(0,94-1,20)$ & 0,310 \\
\hline \multicolumn{3}{|l|}{ Problemas periodontais } \\
\hline Hígido & 1,0 & \\
\hline Cálculo/Sangramento & $1,00(0,95-1,06)$ & 0,934 \\
\hline Bolsa & $0,92(0,84-1,02)$ & 0,096 \\
\hline Outros (sextante excluído) & $1,01(0,91-1,12)$ & 0,881 \\
\hline
\end{tabular}

*Razão de Prevalências (Intervalo de Confiança 95\%), ajustada por todas as variáveis da tabela. ${ }^{*}$ Valor de p obtido pelo teste de Wald 
Tabela 5. Resultado final para a análise multivariada para as características do uso de serviços odontológicos, ajustadas entre si e pelos fatores predisponentes, capacitantes e necessidades de tratamento, Projeto SB-BRASIL 2003.

\begin{tabular}{|c|c|c|}
\hline Variáveis & $\begin{array}{l}\text { RP ajustada* } \\
\text { IC }(95 \%)\end{array}$ & $\begin{array}{l}\text { Valor } \\
\text { de } p^{* *}\end{array}$ \\
\hline \multicolumn{3}{|l|}{ Fatores Predisponentes } \\
\hline \multicolumn{3}{|l|}{ Sexo } \\
\hline Masculino & 1,0 & \\
\hline Feminino & $1,09(1,05-1,13)$ & $<0,001$ \\
\hline \multicolumn{3}{|l|}{ Escolaridade } \\
\hline$\geq 9$ anos & 1,0 & \\
\hline $5-8$ & $1,21(1,14-1,29)$ & $<0,001$ \\
\hline $1-4$ & $1,31(1,22-1,40)$ & $<0,001$ \\
\hline Nenhuma & $1,34(1,23-1,46)$ & $<0,001$ \\
\hline \multicolumn{3}{|l|}{ Fatores Capacitantes } \\
\hline \multicolumn{3}{|l|}{ Renda per capita (em Reais) } \\
\hline Até 50 & 1,0 & \\
\hline $51-100$ & $0,92(0,88-0,96)$ & $<0,001$ \\
\hline $101-200$ & $0,74(0,70-0,79)$ & $<0,001$ \\
\hline$\geq^{\wedge} 201$ & $0,49(0,45-0,54)$ & $<0,001$ \\
\hline \multicolumn{3}{|l|}{ Regiões geográficas } \\
\hline Sudeste & 1,0 & \\
\hline Nordeste & $1,17(1,02-1,35)$ & 0,028 \\
\hline Norte & $1,13(0,97-1,32)$ & 0,111 \\
\hline Sul & $1,18(1,01-1,38)$ & 0,034 \\
\hline Centro-Oeste & $0,94(0,79-1,11)$ & 0,458 \\
\hline \multicolumn{3}{|l|}{ Porte do município } \\
\hline$<10$ mil & 1,0 & \\
\hline $10-100 \mathrm{mil}$ & $0,90(0,82-0,99)$ & 0,026 \\
\hline$>100 \mathrm{mil}$ & $0,84(0,75-0,94)$ & 0,002 \\
\hline \multicolumn{3}{|l|}{ Fatores de necessidade de tratamento } \\
\hline \multicolumn{3}{|c|}{ Necessidade de tratamento autorreferida } \\
\hline Queixa de dor de dente/gengival & 1,0 & \\
\hline Não & $1,05(1,01-1,09)$ & 0,017 \\
\hline \multicolumn{3}{|c|}{$\operatorname{Sim}$} \\
\hline \multicolumn{3}{|c|}{ Necessidade de tratamento diagnosticada pelo profissional } \\
\hline Necessidade de prótese & 1,0 & \\
\hline Não & $1,19(1,11-1,27)$ & $<0,001$ \\
\hline Sim, parcial & $1,31(1,21-1,41)$ & $<0,001$ \\
\hline \multicolumn{3}{|c|}{ Sim, total } \\
\hline \multicolumn{3}{|c|}{ Total de dentes necessitando de tratamento } \\
\hline Nenhum & 1,0 & \\
\hline $1-3$ & $1,06(0,99-1,12)$ & 0,084 \\
\hline 4 ou mais & $1,11(1,03-1,19)$ & 0,004 \\
\hline Não se aplica & $1,08(0,99-1,19)$ & 0,084 \\
\hline \multicolumn{3}{|c|}{ Características da utilização do serviço odontológico } \\
\hline \multicolumn{3}{|c|}{ Motivo da visita } \\
\hline Consulta de rotina & 1,0 & \\
\hline Problemas bucais & $1,17(1,09-1,25)$ & $<0,001$ \\
\hline Outros & $0,93(0,84-1,05)$ & 0,233 \\
\hline \multicolumn{3}{|c|}{ Recebimento de informação sobre saúde bucal } \\
\hline Não & 1,0 & \\
\hline Sim & $1,02(0,98-1,06)$ & 0,418 \\
\hline \multicolumn{3}{|l|}{ Avaliação do atendimento recebido } \\
\hline Bom/Ótimo & 1,0 & \\
\hline Regular & $1,17(1,11-1,24)$ & $<0,001$ \\
\hline Ruim/Péssimo & $1,08(0,95-1,23)$ & 0,252 \\
\hline
\end{tabular}

" RP ajustada IC (95\%): ajustada por todas as variáveis do modelo. " Valor de p obtido pelo teste de Wald 
Pode-se verificar que, após o ajuste, apenas a queixa de dor de dente/gengival ( $\mathrm{RP}=1,07$; IC95\% 1,03-1,11) - entre as necessidades auto-referidas -, a necessidade de prótese parcial $(\mathrm{RP}=1,20$; IC95\% 1,12-1,29) e de prótese total $(\mathrm{RP}=1,35$; IC95\% 1,24-1,48), e a presença de quatro ou mais dentes com necessidade de tratamento $(\mathrm{RP}=1,12$; IC95\% 1,04-1,21) - entre as necessidades definidas pelo profissional -, permaneceram associadas ao evento. A idade, entre as características predisponentes, perdeu a significância estatística.

Os resultados do modelo final de análise hierarquizada são mostrados na Tabela 5. No modelo final, as variáveis que caracterizaram a utilização dos serviços odontológicos foram ajustadas entre si e pelas variáveis dos níveis hierárquicos anteriores. No tocante às variáveis deste bloco distal, o uso de serviço odontológico público foi significativamente maior entre os adultos que procuraram o serviço motivados por algum problema de saúde bucal ( $\mathrm{RP}=1,17$; IC95\% 1,09-1,25) e que avaliaram como regular o atendimento recebido ( $\mathrm{RP}=1,17$; IC95\% 1,11-1,24). O modelo final mostrou ainda que o uso de serviços odontológicos públicos esteve significativa e independentemente associado ao sexo feminino e à menor escolaridade (entre os fatores predisponentes), à menor renda, à residência nas regiões Nordeste e Sul e em municípios de menor porte (entre os fatores capacitantes), à queixa de dor de dente ou gengival e à necessidade de tratamento diagnosticada profissionalmente, no caso, próteses parcial e total e uma maior quantidade de dentes permanentes (entre os fatores de necessidade). As associações mais fortes foram detectadas para as variáveis escolaridade e renda familiar per capita, em ambos os casos, com indicativos de gradiente dose-resposta.

\section{Discussão}

A prevalência do uso de serviços odontológicos públicos verificada entre os adultos brasileiros foi superior ao observado na PNAD-1998 ${ }^{16}$ e no estado da Paraíba ${ }^{17}$. No entanto, é preciso ressalvar que, diferentemente do nosso, os estudos citados abordaram toda a população e não apenas a população adulta, e incluíram na investigação aqueles que não utilizaram qualquer serviço odontológico.

Entre as variáveis predisponentes e capacitantes, que constituem os blocos de variáveis mais distais do modelo hierarquizado, sexo e escolaridade (entre as primeiras), renda, porte do muni- cípio e região geográfica de residência (entre as últimas) mostraram-se no presente estudo, as mulheres utilizaram mais os serviços públicos odontológicos que os homens. Alguns estudos brasileiros evidenciaram uma maior utilização de serviços odontológicos pelas mulheres, mas eles não fizeram distinção entre serviços públicos e privados ${ }^{17-20}$. A maior utilização de serviços odontológicos públicos pelas mulheres pode estar relacionada a uma maior disponibilidade de tempo para comparecer às unidades de saúde pública no horário de funcionamento, em razão das mulheres apresentarem um nível de ocupação (percentual de pessoas ocupadas) mais baixo que os homens ${ }^{21}$.

Em relação aos achados que associaram ao uso de serviços odontológicos públicos a escolaridade e renda mais baixas, é importante ressaltar alguns aspectos. Em primeiro lugar, eles evidenciaram que a população atendida pelos serviços públicos é aquela socioeconomicamente menos favorecida, o que foi também detectado em estudos internacionais ${ }^{22}$ e brasileiros, quer num caráter mais geral ${ }^{23}$, ou específico, no caso, os serviços odontológicos ${ }^{16}$. Isso vai ao encontro das expectativas que vinculam os serviços públicos à equidade na atenção à saúde. Em segundo lugar, o fato das associações observadas para essas variáveis terem sido as de maior magnitude, coloca-as, no presente estudo, como as características mais marcantes na discriminação de usuários de serviços odontológicos públicos e privados. Em terceiro lugar, cabe lembrar que essas características se reforçam mutuamente e frequentemente estão correlacionadas ${ }^{24}$, o que às vezes impõe aos pesquisadores a decisão de não incluí-las simultaneamente no modelo explicativo testado, para evitar a colinearidade ${ }^{25}$. No presente trabalho, elas permaneceram independentemente associadas ao uso do serviço público, com direção e intensidade semelhantes.

A influência da renda sobre o tipo de serviço utilizado é previsível: a opção pelos serviços públicos reflete uma maior dificuldade ou mesmo a incapacidade de custeio dos serviços privados. A importância da renda como determinante do acesso aos serviços odontológicos no Brasil é reforçada na população adulta, pois esta tem sido historicamente desconsiderada pelo setor público na definição das prioridades de atenção à saúde bu$\mathrm{cal}^{16}$. No que diz respeito à escolaridade, ela capacita o indivíduo para atividades que resultem em melhor renda - e por conseguinte - na maior capacidade do custeio dos serviços de saúde. Além disso, ela predispõe o indivíduo a uma maior cons- 
ciência e valorização da saúde, ou seja, possibilita o acesso à informação, favorecendo atitudes e comportamentos que resultam em melhores condições de saúde, tais como autoeficácia, controle e capacidade de enfrentamento dos problemas ${ }^{24}$. Nesse aspecto, os serviços odontológicos públicos estão sendo utilizados por uma parcela da população com menos acesso a informações importantes para a preservação da saúde.

Dados da PNAD-2003 ${ }^{20}$ evidenciaram um acesso a serviços odontológicos (sem distinção de privado e público) diferenciados geograficamente, considerando unidade federativa e localização do domicílio (se urbano ou rural). Nesse mesmo estudo ${ }^{20}$, a utilização de serviços odontológicos esteve associada a variáveis de contexto, no caso, a uma maior razão número de dentistas por mil habitantes e maior oferta de ações de saúde bucal. Para esses autores, esses achados podem ser consequentes de maiores investimentos em saúde, de maior resolutividade das ações de saúde (no caso dos estados), e ao acesso facilitado pela maior oferta de serviços e menor distância deles (no caso da localização do domicílio). Em nosso estudo, encontramos uma maior utilização de serviços públicos nas regiões Nordeste e Sul, comparativamente à região Sudeste, e em municípios de pequeno porte, comparativamente aos de maior porte. É possível que as diferenças regionais na utilização de serviços públicos possam estar relacionadas a diferenças na oferta de serviços. Em 2002, a razão de número de dentistas-SUS por mil habitantes, considerando a população de 35-44 anos, era maior nas regiões Nordeste e Sul, se confrontada com a região Sudeste ${ }^{26}$. Além disso, nessa mesma época, a cobertura do Programa Saúde da Família, que passou a contar com uma equipe de saúde bucal a partir de $2000^{27}$, era maior nessas regiões ${ }^{28}$. Em relação à maior utilização de serviços públicos em municípios de pequeno porte, talvez isso seja influenciado pelo menor poder aquisitivo de sua população e pela menor disponibilidade de serviços odontológicos privados.

As variáveis de necessidade que permaneceram independentemente associadas ao uso de serviços públicos (queixa de dor de dente ou gengival, necessidade de prótese parcial e total, e uma maior quantidade de dentes permanentes demandando tratamento) evidenciam que a população usuária desses serviços apresentava piores condições de saúde bucal e maior necessidade de tratamento que os usuários de serviços privados.

A queixa de dor é um indício da gravidade do problema de saúde bucal que motivou a procura por atendimento. Ela pode refletir a não utilização rotineira de serviços odontológicos ${ }^{11}$ e geralmente essas queixas são atendidas em consultas de urgência. Pacientes odontológicos atendidos em caráter de urgência retornam para novos atendimentos com maior frequência e recebem mais procedimentos cirúrgicos, que representam uma solução pontual dos problemas. Esses pacientes, a longo prazo, apresentarão mais necessidades de tratamento acumuladas, iniciando um ciclo de atendimentos emergenciais que impactam negativamente a sua saúde bucal ${ }^{29}$.

As associações com as variáveis de necessidades definidas ao exame clínico evidenciaram não só que os usuários de serviços públicos demandaram tratamentos curativos e reabilitadores, de maior complexidade, como também que eles apresentavam piores condições de saúde bucal. Esses resultados são consistentes com um padrão de atenção à saúde bucal, observado em estudos internacionais $^{30}$ e nacionais ${ }^{25}$, que vincula os serviços públicos à realização de extrações e procedimentos curativos, em uma clientela com a saúde bucal prejudicada. É possível ainda que essa situação seja um reflexo da histórica desassistência odontológica à população adulta brasileira, restrita àqueles com vínculo empregatício e caracterizada por consultas emergenciais mutiladoras ${ }^{1,3}$.

As características de utilização de serviços odontológicos que permaneceram associados ao uso dos serviços públicos foram as visitas motivadas por um problema de saúde e uma avaliação regular do atendimento recebido. A dicotomia serviços públicos-resolução de problemas e serviços privados-ações preventivas e de manutenção está presente em países desenvolvidos ${ }^{30,31}$ e em diferentes cidades brasileiras ${ }^{9,25}$. Nossos resultados são consoantes com os citados acima, e consistentes com os achados que relacionaram a queixa de dor com a utilização de serviços públicos. Isso pode estar relacionado à organização do acesso aos serviços: no Brasil, preconiza-se que os indivíduos com maior necessidade de tratamento sejam priorizados num primeiro momento de organização da atenção ${ }^{32}$. Atendimentos odontológicos voltados para resolução de problemas acabam por vincular o serviço público a atendimentos emergenciais, com as consequências nefastas já discutidas anteriormente. Além disso, é contrário ao paradigma da boa atenção odontológica, centrada no monitoramento e nas ações preventivas, com baixa frequência de restaurações e extrações ${ }^{30}$.

A pior avaliação dos serviços públicos, comparativamente aos privados, tem paralelo em 
outro estudo brasileiro ${ }^{16}$. A insatisfação dos usuários com o serviço público pode derivar do tempo de espera pelo atendimento, da impossibilidade de escolha do profissional, do não atendimento de todas as necessidades e da desorganização dos processos de trabalho dos profissionais ${ }^{33}$. Por vezes, a insatisfação com o atendimento odontológico público pode estar relacionada mais à diminuição da oferta e à demora em receber o atendimento do que com a avaliação da capacidade técnica do profissional envolvido e dos equipamentos utilizados ${ }^{34}$.

O caráter transversal do presente estudo não permite estabelecer uma relação do tipo causaconsequência entre as covariáveis do modelo e o evento investigado, o que constitui um limite à interpretação dos resultados. Existem também evidências de uma sobrerrepresentação da população feminina no SB-Brasil 2003: 67,6\% da população adulta selecionada eram mulheres, contra uma estimativa de $51,5 \%$ na população adulta brasileira, segundo dados do IBGE também disponíveis na página do Departamento de Informática do SUS - DATASUS ${ }^{26}$, o que pode comprometer a validade externa. Por outro lado, a força do estudo deriva de sua abrangência, da riqueza de dados coletados, que incluem aqueles originados de exames clínicos e dos cuidados metodológicos que lhe conferem validade interna. Ademais, ao nosso conhecimento, este é o primeiro estudo epidemiológico, de abrangência nacional, que compara a utilização de serviços odontológicos públicos e privados, pela população adulta.

Em síntese, os resultados do presente estudo mostraram que a população adulta que demandou os serviços odontológicos públicos é a menos favorecida socioeconomicamente, apresentava maiores necessidades de tratamento e avaliou o atendimento recebido como regular. A situação de grande necessidade acumulada reflete o histórico apartamento desse segmento populacional na atenção à saúde bucal em nosso país e coloca um grande desafio ao SUS, considerando o seu papel na redução das desigualdades e de provimento de acesso universal ao cuidado integral.

O cenário da atenção à saúde bucal no Brasil tem sofrido transformações recentes, como a integração da saúde bucal à Estratégia de Saúde da Família, a criação dos Centros de Especialidades Odontológicas e dos Laboratórios Regionais de Prótese Odontológica, que convivem com ações preventivas em curso há mais tempo, como a fluoretação da água de abastecimento público. A expectativa é que essas transformações promovam uma alteração positiva no quadro de saúde da população adulta brasileira, e que os inquéritos de saúde bucal subsequentes (como o SB-Brasil 2010 recém finalizado) possam nos trazer evidências de mudanças positivas no estado de saúde bucal dessa população, independentemente associadas ao uso de serviços odontológicos públicos.

\section{Colaboradores}

RS Pinto, DL Matos e AI Loyola Filho participaram igualmente de todas as etapas de elaboração do artigo. 


\section{Referências}

1. Narvai PC, Frazão P. Saúde bucal no Brasil: muito além do céu da boca. Rio de Janeiro: Editora Fiocruz; 2008.

2. Nickel DA, Lima FG, Silva BB. Modelos assistenciais em saúde bucal no Brasil. Cad Saude Publica 2008; 24(2):241-246.

3. Groisman S, Moraes NM, Chagas LD. A evolução da atenção à saúde no Brasil: o contexto da saúde bucal. Brasília: Cadernos da ABOPREV II; 2005.

4. Oliveira, AGRC. Levantamentos epidemiológicos em saúde bucal no Brasil. In: Antunes JLF, Peres MA, organizadores. Fundamentos de Odontologia: Epidemiologia da Saúde Bucal. Rio de Janeiro: Guanabara Koogan; 2006. p.32-48.

5. Organização Pan-Americana de Saúde. In: Costa JFR, Chagas LD, Silvestre RM, organizadores. A Política Nacional de Saúde Bucal do Brasil: registro de uma conquista histórica. Brasília: Organização Pan-Americana de Saúde (OPAS); 2006.

6. Andersen RM, Newman JF. Societal and individual determinants of medical care utilization in the United States. Milbank Mem Fund Q 1973; 51(1):95-124.

7. Gilbert HG, Duncan RP, Bruce V. Determinants of dental care use in dentate adults: six-monthly use during a 24-month period in the Florida Dental Care Study. Soc Sci Med 1998; 47(6):727-737.

8. Tennstedt SL, Brambilla DL, Jette AM, McGuire SM. Understanding dental service use by older adults sociobehavioral factors vs. need. J Public Health Dent 1994; 54(4):211-219.

9. Camargo MBJ, Dumith SC, Barros AJD. Uso regular de serviços odontológicos entre adultos: padrões de utilização e tipos de serviços. Cad Saude Publica 2009; 25(9):1894-1906.

10. Matos DL, Lima-Costa MFF, Guerra HL, Marcenes W. Projeto Bambuí: estudo de base populacional dos fatores associados com o uso regular de serviços odontológicos em adultos. Cad Saude Publica 2001; 17(3):661-668.

11. Lacerda JT, Simionato EM, Peres KG, Peres MA, Traebert J, Marcenes W. Dor de origem dental como motivo de consulta odontológica em uma população adulta. Rev Saude Publica 2004; 38(3):453-458.

12. Roncalli AG, Frazão P, Patussi MP, Araújo IC, Ely HC, Batista SM. Projeto SB2000: uma perspectiva para a consolidação da Epidemiologia em Saúde Bucal Coletiva. Rev Bras Odont Saude Coletiva 2000; 1(2):9-25.

13. Brasil. Ministério da Saúde (MS). Projeto SB2000: condições de saúde bucal da população brasileira no ano de 2000. Manual de calibração dos examinadores. Brasília: Ministério da Saúde (MS); 2001.

14. World Health Organization (WHO). Oral Health Surveys: Basic methods. $4^{\text {th }}$ ed. Geneva: ORH/EPID; 1997.

15. Victora CG, Huttly SR, Fuchs SC, Olinto MTA. The role of conceptual frameworks in epidemiological analysis: a hierarquical approach. Int J Epidemiol 1997; 26(1):224-27.

16. Barros AJD, Bertoldi AD. Desigualdades na utilização e no acesso a serviços odontológicos: uma avaliação em nível nacional. Cien Saude Colet 2002; 7(4):709-717.
17. Rocha RACP, Goes PSA. Comparação do acesso aos serviços de saúde bucal em áreas cobertas e não cobertas pela Estratégia Saúde da Família em Campina Grande, Paraíba, Brasil. Cad Saude Publica 2008; 24(12):2871-2880.

18. Araújo CS, Lima RC, Peres MA, Barros, AJD. Utilização de serviços odontológicos e fatores associados: um estudo de base populacional no Sul do Brasil. Cad Saude Publica 2009; 25(5):1063-1072.

19. Manhães ALD, Costa AJL. Acesso a e utilização de serviços odontológicos no Estado do Rio de Janeiro, Brasil, em 1998: um estudo exploratório a partir da Pesquisa Nacional por Amostra de Domicílios. Cad Saude Publica 2008; 24(1):207-218.

20. Pinheiro RS, Torres TZG. Uso de serviços odontológicos entre os Estados do Brasil. Cien Saude Colet 2006; 11(4):999-1010.

21. Instituto Brasileiro de Geografia e Estatística (IBGE). Pesquisa Nacional por Amostra de Domicílios - Síntese de Indicadores 2003. Rio de Janeiro: Instituto Brasileiro de Geografia e Estatística (IBGE); 2004.

22. Nguyen L, Hakkinen U. Choices and utilization in dental care: Public vs. private dental sectors, and the impact of a two-channel financed health care system. Eur J Health Econom 2006; 7(2):99-106.

23. Ribeiro MCSA, Barata RB, Almeida MF, Silva ZP. Perfil sociodemográfico e padrão de utilização de serviços de saúde para usuários e não-usuários do SUS - PNAD 2003. Cien Saude Colet 2006; 11(4):10111022.

24. Mechanic D. Disadvantage, Inequality, And Social Policy. Health Aff 2002; 21(2):48-59.

25. Matos DL, Lima-Costa MF, Guerra HL, Marcenes W. Projeto Bambuí: avaliação de serviços odontológicos privados, públicos e de sindicato. Rev Saude Publica 2002; 36(2):237-243.

26. Brasil. Ministério da Saúde (MS). Departamento de Informática do SUS. Informações de Saúde [acessado 2009 dez 30] [Documento da internet]. Disponível em: http://www2.datasus.gov.br/DATASUS/index. php?area $=02$

27. Brasil. Portaria $n^{\circ}$. 1.444 , de 28 de dezembro de 2000. Estabelece incentivo financeiro para a reorganização da atenção à saúde bucal prestada nos municípios por meio do Programa de Saúde da Família. Diário Oficial da União 2000; 29 dez.

28. Brasil. Ministério da Saúde [homepage na internet]. Consolidado histórico de cobertura da Saúde da Família 2003 [acessado 2009 dez 30] [Documento da internet]. Disponível em: http://dtr2004.saude. gov.br/dab/docs/abnumeros/historico_2003.pdf

29. Luzzi L, Spencer AJ. Public dental service utilization among adults in South Australia. Aust Dent J 2009; 54(2):154-160.

30. Brennan DS, Luzzi L, Roberts-Thomson KF. Dental service patterns among private and public adult patients in Australia. BMC Health Serv Res [periódico na Internet] 2008 jan [acessado $2011 \mathrm{dez} 11$ ]; 8:1. Disponível em: http://www.biomedcentral.com/ $1472-6963 / 8 / 1$ 
31. Roberts-Thomson KF, Luzzi L, Brennan DS. Social inequality in use of dental services: relief of pain and extractions. Aust N Z J Public Health 2008; 32(5):444-449.

32. Brasil. Ministério da Saúde (MS). Normas e Manuais Técnicos. Caderno de Atenção Básica no.17: Saúde Bucal. Brasília: Ministério da Saúde (MS); 2006.

33. Gouveia GC, Souza WV, Luna CF, Souza-Júnior PRB, Szwarcwald CL. Health care users' satisfaction in Brazil, 2003. Cad Saude Publica 2005; 21(Supl.) 1:S109-S118.

34. Hancock M, Calnan M, Manley G. Private or NHS General Dental Service care in the United Kingdom? A study of public perception and experience. J Public Health Med 1999; 21(4):415-420.

Artigo apresentado em 21/03/2010

Aprovado em 20/04/2010

Versão final apresentada em 19/05/2010 